\title{
Some results of Watson, Plancherel type integral transforms related to the Hartley, Fourier convolutions and applications
}

\author{
Tuan $\operatorname{Trinh}^{1}$ \\ ${ }^{1}$ Electric Power University
}

September 25, 2021

\begin{abstract}
In this work, we study the Watson-type integral transforms for the convolutions related to the Hartley and Fourier transformations. We establish necessary and sufficient conditions for these operators to be unitary in the L 2 (R) space and get their inverse represented in the conjugate symmetric form. Furthermore, we also formulated the Plancherel-type theorem for the aforementioned operators and prove a sequence of functions that converge to the original function in the defined L 2 (R) norm. Next, we study the boundedness of the operators ( $\mathrm{T} \mathrm{k}$ ). Besides, showing the obtained results, we demonstrate how to use it to solve the class of integro-differential equations of Barbashin type, the differential equations, and the system of differential equations. And there are numerical examples given to illustrate these.
\end{abstract}

\section{Hosted file}

Some results of Watson, Plancherel type integral transforms_and.pdf available at https: //authorea.com/users/437772/articles/539176-some-results-of-watson-plancherel-type-

integral-transforms-related-to-the-hartley-fourier-convolutions-and-applications 